\title{
On the Control of End Point Tensions in a Vibration Problem
}

\author{
Murat Subaşi, Hakki Güngör, and Seda Iğret Araz
}

\begin{abstract}
This study aims to investigate the optimality conditions in an optimal control problem governed by a hyperbolic problem. In the problem of vibration of one dimensional wire, the minimization of the distance between the end points of wire and the desired target functions is carried out by controlling the tensions of end points.
\end{abstract}

Index Terms-Optimal control, optimal solutions, second-order hyperbolic equations.

\section{INTRODUCTION}

This paper deals with the problem

$$
J_{\alpha^{*}}=\inf _{h \in H} J_{\alpha}(h)=J_{\alpha}\left(h^{*}\right)
$$

By the functional

$$
\begin{aligned}
J_{\alpha}(h) & =\beta_{1} \int_{0}^{T}\left[u(0, t)-y_{1}(t)\right]^{2} d t+\beta_{2} \int_{0}^{T}\left[u(l, t)-y_{2}(t)\right]^{2} d t \\
& +\alpha\|h\|_{H}^{2}
\end{aligned}
$$

On the admissible pairs set

$$
h(t) \in H:=\left\{h=\{f(t) ; g(t)\}:\|h(t)\|_{H}^{2} \leq \tilde{h}\right\}
$$

Subject to the following one dimensional vibration problem on the domain $\Omega:=(0, l) \times(0, T)$;

$$
\begin{gathered}
p(x) u_{t t}=\left(k(x) u_{x}\right)_{x}+F(x, t), \quad(x, t) \in \Omega \\
u(x, 0)=\varphi_{1}(x), u_{t}(x, 0)=\varphi_{2}(x), x \in(0, l) \\
k(0) u_{x}(0, t)=f(t), k(l) u_{x}(l, t)=g(t), \quad t \in(0, T)
\end{gathered}
$$

Here, the coefficient functions $p(x)$ and $k(x)$ (Young modulus) represent the density and rigidity of an one dimensional wire respectively and satisfy the following conditions

$$
0<p_{0} \leq p(x) \leq p_{1}, \quad 0<k_{0} \leq k(x) \leq k_{1}, \quad \stackrel{\circ}{\forall} x \in(0, l)
$$

Also, the initial data represent the initial status and initial velocity of the wire and taken from the spaces

$$
\varphi_{1}(x) \in H^{1}(0, l), \varphi_{2}(x) \in L_{2}(0, l) .
$$

Manuscript received October 10, 2016; revised April 13, 2017.

Murat Subaşı, Hakkı Güngör, Seda İğret Araz are with the Ataturk University, Science Faculty, Department of Mathematics, 25240, Erzurum, Turkey (e-mail: msubasi@ atauni.edu.tr, hakki.gungor11@ogr.atauni.edu.tr, seda.araz@ atauni.edu.tr).
The functions $f(t)$ and $g(t)$ mean the tensions (Hooke Law) at the end points and play the role of control functions.

The inner product and norm on the set $H$, which is sub space of $L_{2}(0, T) \times L_{2}(0, T)$, can be defined as

$$
\left\langle h_{1}, h_{2}\right\rangle_{H}=\int_{0}^{T}\left[f_{1}(t) f_{2}(t)+g_{1}(t) g_{2}(t)\right] d t
$$

and

$$
\|h(t)\|_{H}^{2}=\int_{0}^{T}\left[f^{2}(t)+g^{2}(t)\right] d t
$$

For $\quad \forall h_{1}:=\left\{f_{1}, g_{1}\right\} \in H, \forall h_{2}:=\left\{f_{2}, g_{2}\right\} \in H \quad$ and $\forall h:=\{f, g\} \in H$.

We are interested in the weak solution $u \in H^{1}(\Omega)$ with $u(x, 0)=\varphi_{1}(x)$ and $u_{t}(x, 0)=\varphi_{2}(x), x \in(0, l)$ of the problem (4)-(6) which satisfies the following integral equation;

$$
\begin{aligned}
& \int_{\Omega}\left[-p(x) u_{t} \eta_{t}+k(x) u_{x} \eta_{x}\right] d x d t-\int_{0}^{T} g(t) \eta(l, t) d t \\
& +\int_{0}^{T} f(t) \eta(0, t) d t-\int_{0}^{T} \int_{0}^{l} F(x, t) \eta(x, t) d x d t=0
\end{aligned}
$$

For $\forall \eta \in H^{1}(\Omega), \quad \eta(x, T)=0$.

It can be seen in [1] that solution in the sense of (9) is exist, unique and satisfies the inequalities

$$
\begin{array}{r}
\max _{0 \leq t \leq T}\left(\|u(\cdot, t)\|_{L_{2}(0, l)}^{2}+\left\|u_{t}(\cdot, t)\right\|_{L_{2}(0, l)}^{2}+\left\|u_{x}(\cdot, t)\right\|_{L_{2}(0, l)}^{2}\right) \\
\leq c_{0}\left(\left\|\varphi_{1}\right\|_{H^{1}(0, l)}^{2}+\left\|\varphi_{2}\right\|_{L_{2}(0, l)}^{2}+\|F\|_{L_{2}(\Omega)}^{2}+\|h\|_{H}^{2}\right)
\end{array}
$$

and

$$
\|u\|_{H^{1}(\Omega)}^{2} \leq c_{1}\left(\left\|\varphi_{1}\right\|_{H^{1}(0, l)}^{2}+\left\|\varphi_{2}\right\|_{L_{2}(0, l)}^{2}+\|F\|_{L_{2}(\Omega)}^{2}+\|h\|_{H}^{2}\right)
$$

where $c_{0}$ and $c_{1}$ are independent from $\varphi_{1}, \varphi_{2}, F$ and $h$.

The functional $J_{\alpha}(h)$ is called cost functional with the given numbers $\beta_{1}$ and $\beta_{2}$ such as $\beta_{1} \geq 0, \beta_{2} \geq 0, \beta_{1}+\beta_{2} \neq 0$. The functions $y_{1}(t) \in L_{2}(0, T)$ and $y_{2}(t) \in L_{2}(0, T)$ are given functions. The number $\alpha>0$ 
is regularization parameter and the third term in the cost functional is called regularization term; its role is to avoid using "too large" controls in the minimization of $J_{\alpha}(h)$.

So, we want to control the tensions of end points in an one-dimensional vibration problem, given by the functions $f(t)$ and $g(t)$, in order to minimize the distance between the end points $u(0, t), u(l, t)$ and desired target functions $y_{1}(t), y_{2}(t)$.

In [2]-[17], similar problems with different controls and cost functions have been investigated by some authors.

This study is contributive in view of the property of controllability of left and right end point tensions simultaneously.

The main issues we deal with in the considered optimal control problem are:

1) Establishing existence and uniqueness of an optimal pair $\left\{u^{*}, h^{*}\right\}$.

2) Deriving necessary and sufficient optimality conditions.

3) Constructing an algorithm for the numerical approximation of $\left\{u^{*}, h^{*}\right\}$.

\section{EXISTENCE AND UNIQUENESS OF AN OPTIMAL PAIR}

The strategy to prove existence and uniqueness of an optimal control is to use the relationship between minimization of quadratic functional and variational problems corresponding to symmetric bilinear forms.

Principally, the key point is to rewrite the functional $J_{\alpha}(h)$ in the following way:

$$
J_{\alpha}(h)=b(h, h)-2 L h+q
$$

where

$$
\begin{gathered}
b(h, h)=\beta_{1} \int_{0}^{T}[u(0, t ; h)-u(0, t ; 0)]^{2} d t \\
+\beta_{2} \int_{0}^{T}[u(l, t ; h)-u(l, t ; 0)]^{2} d t+\alpha\|h\|_{H}^{2}, \\
L h=\beta_{1} \int_{0}^{T}[u(0, t ; h)-u(0, t ; 0)]\left[y_{1}(t)-u(0, t ; 0)\right] d t \\
+\beta_{2} \int_{0}^{T}[u(l, t ; h)-u(l, t ; 0)]\left[y_{2}(t)-u(l, t ; 0)\right] d t \\
\text { and } \beta_{1} \int_{0}^{T}\left[y_{1}(t)-u(0, t ; 0)\right]^{2} d t+\beta_{2} \int_{0}^{T}\left[y_{2}(t)-u(l, t ; 0)\right]^{2} d t .
\end{gathered}
$$

Here $b(h, h)$ is a bilinear, symmetric, continuous and coercive and $L$ is a linear, continuous functional in $H$.

Then, since the conditions of following theorem given by Lions [18] are hold, the existence and uniqueness of the optimal solution is assured.

Theorem: Let $b\left(h^{*}, h\right)$ continuous symmetric bilinear form with coercive property on $H$. Then there exists a unique element $h^{*} \in H$ such that

$$
J_{\alpha}\left(h^{*}\right)=\inf _{h \in H} J_{\alpha}(h) .
$$

\section{LAGRANGE MULTIPLIERS AND OPTIMALITY CONDITIONS}

To obtain optimality conditions, let us write the augmented functional

$$
\begin{aligned}
\tilde{J}_{\alpha}(h) & =\beta_{1} \int_{0}^{T}\left[u(0, t)-y_{1}(t)\right]^{2} d t+\beta_{2} \int_{0}^{T}\left[u(l, t)-y_{2}(t)\right]^{2} d t \\
& +\alpha\|h\|_{H}^{2}+\int_{\Omega} \eta\left[p(x) u_{t t}-\left(k(x) u_{x}\right)_{x}-F(x, t)\right] d x d t .
\end{aligned}
$$

The Euler equation for this augmented functional will be in the form of

$$
\begin{aligned}
\tilde{J}_{\alpha}^{\prime}\left(h^{*}\right) \delta h & =\int_{0}^{T}\left[2 \beta_{1}\left(u^{*}(0, t)-y_{1}(t)\right)-k(0) \eta_{x}(0, t)\right] \delta u^{*}(0, t) d t \\
& +\int_{0}^{T}\left[2 \beta_{2}\left(u^{*}(l, t)-y_{2}(t)\right)+k(l) \eta_{x}(l, t)\right] \delta u^{*}(l, t) d t \\
& +\int_{\Omega}\left[p(x) \eta_{t t}-\left(k(x) \eta_{x}\right)_{x}\right] \delta u^{*} d x d t \\
& +\int_{0}^{l} p(x) \eta(x, T) \delta u_{t}^{*}(x, T) d x-\int_{0}^{l} p(x) \eta_{t}(x, T) \delta u^{*}(x, T) d x \\
& +\int_{0}^{T}\left[\eta(0, t)+2 \alpha f^{*}\right] \delta f d t+\int_{0}^{T}\left[-\eta(l, t)+2 \alpha g^{*}\right] \delta g d t .
\end{aligned}
$$

Now, we consider the following adjoint boundary-value problem;

$$
\begin{gathered}
p(x) \eta_{t t}^{*}-\left(k(x) \eta_{x}^{*}\right)_{x}=0 \\
k(0) \eta_{x}^{*}(0, t)=2 \beta_{1}\left(u^{*}(0, t)-y_{1}(t)\right) \\
k(l) \eta_{x}^{*}(l, t)=-2 \beta_{2}\left(u^{*}(l, t)-y_{2}(t)\right) \\
\eta^{*}(x, T)=\eta_{t}^{*}(x, T)=0 .
\end{gathered}
$$

By the solution of adjoint boundary-value problem, we mean the function $\eta^{*} \in H^{1}(\Omega)$ which satisfies the following integral identity;

$$
\begin{aligned}
& \int_{0}^{T} \int_{0}^{l}\left[p(x) \eta_{t}^{*} \phi_{t}-k(x) \eta_{x}^{*} \phi_{x}\right] d x d t=2 \beta_{1} \int_{0}^{T}\left[u^{*}(0, t)-y_{1}(t)\right] \phi(0, t) d t \\
& +2 \beta_{2} \int_{0}^{T}\left[u^{*}(l, t)-y_{2}(t)\right] \phi(l, t) d t-\int_{0}^{l} p(x) \eta_{t}^{*}(x, 0) \phi(x, 0) d x
\end{aligned}
$$

for $\forall \phi \in H^{1}(\Omega)$.

Using the adjoint boundary-value problem the Euler equation becomes

$$
\begin{aligned}
\tilde{J}_{\alpha}^{\prime}\left(h^{*}\right) \delta h & =\int_{0}^{T}\left[\eta^{*}(0, t)+2 \alpha f^{*}\right] \delta f d t \\
& +\int_{0}^{T}\left[-\eta^{*}(l, t)+2 \alpha g^{*}\right] \delta g d t, \quad \forall \delta h \in H .
\end{aligned}
$$

Theorem: The control $h^{*}=\left\{f^{*}, g^{*}\right\}$ and the state 
$u^{*}=u\left(h^{*}\right)$ are optimal if and only if there exists a multiplier $\eta^{*}$ such that $h^{*}, u^{*}$ and $\eta^{*}$ satisfy the following optimality conditions;

$$
\left\{\begin{array}{c}
p(x) u_{t t}^{*}=\left(k(x) u_{x}^{*}\right)_{x}+F(x, t) \\
u^{*}(x, 0)=\varphi_{1}(x), u_{t}^{*}(x, 0)=\varphi_{2}(x) \\
k(0) u_{x}^{*}(0, t)=f(t), \quad k(l) u_{x}^{*}(l, t)=g(t) \\
p(x) \eta_{t t}^{*}-\left(k(x) \eta_{x}^{*}\right)_{x}=0 \\
k(0) \eta_{x}^{*}(0, t)=2 \beta_{1}\left(u^{*}(0, t)-y_{1}(t)\right) \\
k(l) \eta_{x}^{*}(l, t)=-2 \beta_{2}\left(u^{*}(l, t)-y_{2}(t)\right) \\
\eta^{*}(x, T)=\eta_{t}^{*}(x, T)=0 \\
\eta^{*}(0, t)+2 \alpha f^{*}=0,-\eta^{*}(l, t)+2 \alpha g^{*}=0
\end{array}\right.
$$

The term $\left\{\eta^{*}(0, t)+2 \alpha f^{*},-\eta^{*}(l, t)+2 \alpha g^{*}\right\}$ is called the gradient of $J_{\alpha}(h)$ at $h^{*}$ and denoted by the symbol $\nabla J_{\alpha}\left(h^{*}\right)$.

\section{CONSTRUCTING AN ITERATIVE AlgORITHM FOR NUMERICAL APPROXIMATION}

In this section, we give an iterative procedure by the method of projection of the gradient to compute a sequence of controls $\left\{h_{k}\right\}$, convergent to the optimal solution $h^{*}$.

Select an initial control $h_{0} \in H$. If $h_{k}$ is known $(k \geq 0)$, then $h_{k+1}$ is computed according to the following scheme.

1) Solve the integral equation of (9) in order to obtain the generalized solution $u_{k}$.

2) Using $u_{k}$, solve the adjoint equation (14) and find $\eta_{k}$. 3) Set

$$
h_{k+1}=P_{H}\left\lfloor h_{k}-\mu_{k} \nabla J_{\alpha}\left(h_{k}\right)\right\rfloor
$$

where $P_{H}\left\lfloor h_{k}-\mu_{k} \nabla J_{\alpha}\left(h_{k}\right)\right\rfloor$ is the projection of the element $h_{k}-\mu_{k} \nabla J_{\alpha}\left(h_{k}\right)$ on the set $H$.

4) Select the small enough relaxation parameter $\mu_{k}>0$ such that

$$
J_{\alpha}\left(h_{k+1}\right)<J_{\alpha}\left(h_{k}\right)
$$

We refer to the study [12] for the proof of the convergence of the minimizing sequence $\left\{h_{k}\right\}$ to the optimal solution $h^{*}$.

\section{CONCLUSION}

In the problem of vibration of one dimensional wire, the minimization of the distance between the end points of wire and the desired target functions can be carried out by using the chosen cost functional.

The obtained gradient allows the usage of the method of projection of gradient which converges to the unique optimal solution.

\section{REFERENCES}

[1] O. A. Ladyzhenskaya, Boundary Value Problems in Mathematical Physics, Springer-Verlag, New York, 1985.

[2] A. Bamberger, G. Chavent, and P. Lailly, "About the stability of the inverse problem in 1-D wave equations, application to the interpretation of seismic profiles," Applied Mathematics and Optimization, vol. 5, pp. 1-47, 1979.

[3] J. L. Lions, "Exact controllability, stabilization and perturbations for distributed systems," SIAM Rev., vol. 30, pp. 1-68, 1988.

[4] M. Yamamoto, "Stability, reconstruction formula and regularization for an inverse source hyperbolic problem by a control method," Inverse Problems, vol. 11, no. 2, pp. 481-496, 1995.

[5] S. A. Yu. and I. V. Viktorova, "Numerical reconstitution of a nonlinear source in a hyperbolic equation," Numerical Functional Analysis and Optimization, vol. 25, pp. 137-150, 2004.

[6] B. S. Mordukhovich and J. P. Raymond, "Dirichlet boundary control of hyperbolic equations in the presence of state constraints," Appl. Math. Optim., vol. 49, pp. 145-157, 2004.

[7] R. Cipolattiand and I. F. Lopez, "Determination of coefficients for a dissipative wave equation via boundary measurements," Journal of Mathematical Analysis and Applications, vol. 306, pp. 317-329, 2005.

[8] C. Clason, "A direct method for the numerical time reversal of waves in a heterogeneous medium," Ph.D. Thesis, Technische Universitat München, Zentrum Mathematik, München, 2006.

[9] M. Majewski, "Stability analysis of an optimal control problem for a hyperbolic equation," Journal of Optimization Theory and Applications, vol. 141, pp. 127-146, 2009.

[10] T. Yeloğlu and M. Subaşı, "Simultaneous control of the source terms in a vibrational string problem," Iranian Journal of Science \& Technology, Transaction A, vol. 34, no. A1, 2010.

[11] A. Kröner, "Adaptive finite element methods for optimal control of second order hyperbolic equations, computational methods in applied mathematics," vol. 11, no. 2, pp. 214-240, 2011.

[12] R. K. Tagiyev, "On optimal control of the hyperbolic equation coefficients, automation and remote control," vol. 73, no. 7, pp 1145-1155, 2012.

[13] A. Kowalewski, I. Lasiecka, and J. Sokolowski, Sensitivity Analysis of Hyperbolic Optimal Control Problems, Computational Optimization and Applications, pp. 147-179, 2012.

[14] S. Serovajsky, "Optimal control for systems described by hyperbolic equation with strong nonlinearity," Journal of Applied Analysis and Computation, vol. 3, no. 2, pp. 183-195, 2013.

[15] M. Herty, A. Kurganov, and D. Kurochkin, 'Numerical method for optimal control problems governed by nonlinear hyperbolic systems of pdes," Communications in Mathematical Sciences, vol. 12, no. 50, 2014.

[16] A. Hasanov, "Simultaneous determination of the source terms in a linear hyperbolic problem from the final over determination: Weak solution approach," Journal of Applied Mathematics, pp. 1-19, 2008.

[17] A. Hasanov and B. Mukanova, "Relationship between representation formulas for unique regularized solutions of inverse source problems with final overdetermination and singular value decomposition of input-output operators," IMA Journal of Applied Mathematics, 2014.

[18] J. L. Lions, "Optimal control of systems governed by partial differential equations," Springer-Verlag, New York, 1971.

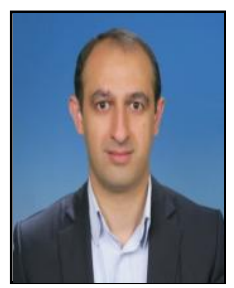

Murat Subași was born in Erzurum, Turkey in 1976 He received his B.S. degree in mathematics from Faculty of Science, Atatürk University in 1996 and the M.Sc. in applied mathematics from Graduate School of Natural and Applied Sciences, Atatürk University in 1999 . He has made the PhD in applied mathematics in 2001

Now he is currently working as professor at Atatürk University. He is interested in optimal control, differential equations theory and numerical optimization. 


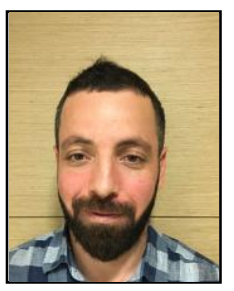

Hakkı GüNgör was born in Ankara, Turkey in 1981 He received his B.S. degree in mathematics from Faculty of Science, Gazi University in 2006 and the M.Sc. in applied mathematics from Graduate School of Natural and Applied Sciences, Karadeniz Technical University in 2009. He has made the PhD in applied mathematics.

Now he is currently working as mathematics teacher at Yedi İklim Academy. He is interested in partial differential equations, optimal control, mathematical modeling and optimization.

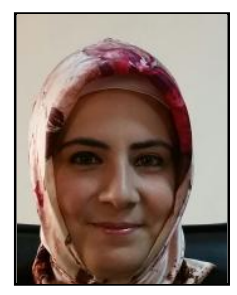

Seda Iğret Araz was born in Sanlıurfa, Turkey in 1987. She received her B.S. degree in mathematics from Faculty of Science, Ataturk University in 2010 and the M.Sc. in applied mathematics from Graduate School of Natural and Applied Sciences, Ataturk University in 2012. She has made the PhD in applied mathematics.

Now she is currently working as research assistant at Gaziantep University, Faculty of Arts and Sciences, Department of Mathematics. She is interested in partial differential equations, optimal control, numerical analysis, mathematical modeling and optimization. 